\title{
Hsp70 inducer, 17-allylamino-demethoxygeldanamycin, provides neuroprotection via anti-inflammatory effects in a rat model of traumatic brain injury
}

\author{
YOUQUAN GU ${ }^{1}$, JUN CHEN ${ }^{2}$, TIANHONG WANG ${ }^{2}, \mathrm{CHAONING} \mathrm{ZHOU}^{1}$, ZHAODONG LIU $^{1}$ and LANHUA MA ${ }^{1}$ \\ ${ }^{1}$ Department of Neurology, Donggang Branch of The First Hospital of Lanzhou University; \\ ${ }^{2}$ Department of Neurology, The First Hospital of Lanzhou University, Lanzhou, Gansu 730000, P.R. China
}

Received October 15, 2015; Accepted April 4, 2016

DOI: $10.3892 /$ etm.2016.3821

\begin{abstract}
Traumatic brain injury (TBI) is the predominant cause of mortality in young adults and children living in China. TBI induces inflammatory responses; in addition, tumor necrosis factor- $\alpha$ (TNF- $\alpha$ ), interleukin-1 $\beta$ (IL-1 $\beta$ ), and IL-6 are important pro-inflammatory cytokines. Considering the observation that Hsp-70 overexpression can exert neuroprotection, identifying a drug that is able to induce the upregulation of Hsp70 has the potential to be a promising therapy for the treatment of neurological diseases. Thus, the present study assessed the clinical effectiveness of an anticancer drug and Hsp70 activator, 17-allylamino-demethoxygeldanamycin (17-AAG), to evaluate its potential as a treatment for patients with TBI. The aim of present study was to determine the neuroprotective effects of 17-AAG following trauma and to investigate the underlying mechanisms of action. To establish rat models, rats were subjected to a controlled cortical impact injury and randomly divided into vehicle or 17-AAG groups. In the 17-AAG group, rats were administered with an intraperitoneal injection of $17-\mathrm{AAG}(80 \mathrm{mg} / \mathrm{kg})$ immediately following the establishment of TBI. The motor function was measured using Neurologic Severity Score, and neuronal death was evaluated using immunofluorescence. The expression levels of GLT-1, Bcl-2 and Hsp-70 were detected by western blot analysis and the expression levels of inflammatory cytokines were quantified using ELISA. The present study determined that 17-AAG
\end{abstract}

Correspondence to: Professor Jun Chen, Department of Neurology, The First Hospital of Lanzhou University, 1 Donggangxi Road, Lanzhou, Gansu 730000, P.R. China

E-mail: guyq@lzu.edu.cn

Abbreviations: 17-AAG, 17-allylamino-demethoxygeldanamycin; TBI, traumatic brain injury; CCI, controlled cortical impact; NSS, Neurologic Severity Score; NeuN, neuron-specific nuclear protein; TNF- $\alpha$, tumor necrosis factor- $\alpha$; IL-6, interleukin-6; IL-1 $\beta$, interleukin-1 $\beta$; GLT-1, glutamate transporter-1

Key words: 17-allylamino-demethoxygeldanamycin, neuroprotection, traumatic brain injury, inflammatory cytokines, neuronal death, rats significantly reduced brain edema and motor neurological deficits $(\mathrm{P}<0.05)$, in addition to increasing neuronal survival. The aforementioned findings are associated with a downregulation of the expression levels of pro-inflammatory cytokines TNF- $\alpha$, IL-1 $\beta$ and IL-6. Conversely, no significant changes of glutamate transporter-1 expression were observed. The present results suggest that 17-AAG treatment may provide a neuroprotective effect by reducing inflammation following TBI.

\section{Introduction}

Traumatic brain injury (TBI) is the leading cause of mortality in young adults and children living in China (1). Head trauma has numerous consequences in the brain, including axonal damage, microvascular alterations and blood-brain barrier disruption (2). The aforementioned outcomes are a result of both primary and secondary mechanisms following injury (3). Primary damage is a result of mechanical factors occurring immediately following trauma. Conversely, the fundamental mechanisms underlying secondary damage in TBI include inflammation, oxygen free radicals, brain edema formation and neuronal apoptosis (4). In particular, previous studies have demonstrated that tumor necrosis factor- $\alpha$ (TNF- $\alpha$ ), interleukin (IL)-1 $\beta$ and IL- 6 are crucial pro-inflammatory cytokines following trauma (5-7). Although the potential effects of TNF- $\alpha$, IL-1 $\beta$ and IL- 6 in human patients with TBI have yet to be elucidated, evidence from animal models has revealed that upregulated expression levels of the aforementioned cytokines are harmful, whereas their attenuation may alleviate tissue damage as well as brain edema, thereby improving the functional outcomes of patients with TBI (8).

Heat shock protein 70 (Hsp70; $72 \mathrm{kDa})$ is a highly stress-inducible member of a chaperone family of proteins (9). Previous studies in focal and global cerebral ischemia models have revealed that the overexpression of Hsp70 is protective against secondary damage in TBI, such as inflammation, oxygen free radicals and brain edema $(10,11)$. Conversely, downregulated expression levels of Hsp70 resulted in a worse outcome in a rat model of stroke (12). Kim et al (13) demonstrated that Hsp70 contributed to the modulation of immune responses by potentiating or inhibiting them. 
It is widely established that inflammation is capable of inducing brain tissue damage following TBI. Thus, the present study aimed to determine whether the Hsp70 activator, 17-allylamino-demethoxygeldanamycin (17-AAG), is able to upregulate the expression levels of Hsp70 in addition to reducing the expression levels of pro-inflammatory cytokines TNF- $\alpha$, IL- $1 \beta$ and IL- 6 , in a rat model of TBI, as observed in neuroprotective processes.

\section{Materials and methods}

Animals and ethics statement. Male Sprague-Dawley rats ( $\mathrm{n}=90$; weight, 280-320 g) obtained from the Lanzhou University Experimental Animal Center (Lanzhou, China) were provided ad libitum access to food and water prior to surgery under optimal conditions (12-h light/dark cycle; $22^{\circ} \mathrm{C}$ ). All procedures were approved by the local legislation for ethics regarding experiments on animals (Ethics Committee of Lanzhou University, Lanzhou, China). The rats were anaesthetized with sodium pentobarbital (Bio-Rad Laboratories, Inc., Shanghai, China) by intraperitoneal injection $(50 \mathrm{mg} / \mathrm{kg}$; Beijing, China).

Models of TBI. A previously described controlled cortical impact (CCI) injury procedure was utilized (14).

Groups and drug administration. Rats were randomly assigned to the vehicle group $(n=30)$ or the 17-AAG group $(n=30)$. The vehicle group received only equal volumes of $0.9 \%$ saline solution $(20 \mathrm{mg} / \mathrm{ml})$ and the $17-\mathrm{AAG}$ group received $17-\mathrm{AAG}$ dissolved in $0.9 \%$ saline that had been stored at $4^{\circ} \mathrm{C}$. Following the establishment of TBI, 17-AAG was immediately administered by a single intraperitoneal injection in the 17-AAG group ( $80 \mathrm{mg} / \mathrm{kg}$ body weight).

Evaluation of brain edema. Brain edema was evaluated by analysis of brain water content, as previously described (15).

Recovery of motor function. Motor function was evaluated as described previously (15). Neurologic Severity Score (NSS) was evaluated at 1, 3,5 and 7 days (15).

Immunofluorescence. Rats were anesthetized with sodium pentobarbital (i.p; $50 \mathrm{mg} / \mathrm{kg}$; Beijing, China) prior to sacrifice. Coronal sections $(10 \mu \mathrm{m})$ were obtained from the anterior area of the left hemisphere. Immunofluorescence was performed as described previously (15). Briefly, brain tissue samples were fixed in 4\% paraformaldehyde (Bio-Rad Laboratories, Inc.) for $24 \mathrm{~h}$, then transferred to a $30 \%$ sucrose solution containing $0.1 \mathrm{~mol} / 1$ phosphate-buffered saline (PBS; $\mathrm{pH}$ 7.4). Incisions were made $200 \mu \mathrm{m}$ apart, from the anterior to the posterior cortex (bregma -1.90 to $-3.00 \mathrm{~mm}$ ) in TBI rats then embedded in optimal cutting temperature compound. Frozen tissue sections $(15 \mu \mathrm{m})$ were cut using a microtome, treated with $0.4 \%$ Triton-100 for 10 min and blocked in normal donkey serum (both Bio-Rad Laboratories, Inc.) for $1 \mathrm{~h}$. The frozen tissue sections were then incubated with mouse anti-neuron-specific nuclear protein (NeuN) polyclonal antibody (diluted 1:100; cat no. sc-33684; Bio-Rad Laboratories, Inc.) overnight at $4^{\circ} \mathrm{C}$. The following day, the tissue sections were incubated with anti-mouse immunoglobulin $\mathrm{G}$ ( $\mathrm{IgG}$ ) secondary antibody (diluted 1:1,000; Santa Cruz Biotechnology, Inc. (Dallas, TX, USA) for $2 \mathrm{~h}$ at $37^{\circ} \mathrm{C}$ in darkness. Images were captured using a laser scanning confocal microscope (Olympus Corp. BX41-P, Tokyo, Japan). PBS was used in place of primary antibodies in the negative control group.

Immunohistochemistry. Rats were anesthetized and sacrificed by intracardiac perfusion with $0.1 \mathrm{~mol} / \mathrm{l} \mathrm{PBS}$ ( $\mathrm{pH} 7.4$ ). The brains was rapidly isolated. Coronal sections $(10 \mu \mathrm{m})$ were obtained from the anterior area of the left hemisphere, washed with $0.1 \mathrm{M}$ PBS, fixed in $10 \%$ formalin (Bio-Rad Laboratories, Inc.) for $24 \mathrm{~h}$ at room temperature, dehydrated in graded ethanol (70, 90, 95 and 100\%), and embedded in paraffin (Bio-Rad Laboratories, Inc.). Tissue sections (6-mm thick) were incubated with an anti-Hsp70 antibody (diluted 1:500; Bio-rad Laboratories, Inc.) at $4^{\circ} \mathrm{C}$ for $24 \mathrm{~h}$. The tissue sections were then incubated with the relevant secondary antibodies for $90 \mathrm{~min}$ at room temperature. Slides were developed with diaminobenzidine substrate (Bio-Rad Laboratories, Inc.) and the images were captured using a microscope (BX41-P; Olympus Corp.).

Western blot analysis. Western blot analysis was performed as previously described (15). Briefly, rats were anesthetized and underwent intracardiac perfusion with $0.1 \mathrm{~mol} / \mathrm{l} \mathrm{PBS}$ ( $\mathrm{pH}$ 7.4). The cortex region of the brains was rapidly isolated, homogenized (BestBio Biotechnology Co., Ltd., Shanghai, China), total proteins were extracted and protein concentration was determined using a Bicinchoninic Acid Protein Assay kit (Bio-Rad Laboratories, Inc.) according to the manufacturer's protocol. The protein samples were subjected to SDS-PAGE (Bio-Rad Laboratories, Inc). Protein concentration was determined using a bicinchoninic acid assay (Beijing Solarbio Science \& Technology Co., Ltd., Beijing, China). Total protein $(35 \mu \mathrm{g})$ was separated by $20 \%$ SDS-PAGE gel electrophoresis ( $80 \mathrm{~V}$ for $30 \mathrm{~min}$ at $28^{\circ} \mathrm{C}$ for spacer gel, and then $120 \mathrm{~V} / 1$ for $1 \mathrm{~h}$ at $28^{\circ} \mathrm{C}$ for separation gel) and transferred from the gel onto polyvinylidene fluoride membranes (Roche Diagnostics $\mathrm{GmbH}$, Mannheim, Germany). The membranes were blocked with $5 \%$ non fat dry milk for $1 \mathrm{~h}$ at room temperature. Following blocking, the membranes were incubated with the following primary antibodies, all purchased from all Santa Cruz Biotechnology, Inc., overnight at $4^{\circ} \mathrm{C}$ : Rabbit anti-Hsp70 polyclonal antibody (diluted 1:500; cat no. sc-33575), rabbit anti-caspase-3 polyclonal antibodies diluted 1:500, rabbit anti-B-cell lymphoma 2 (Bcl-2) polyclonal antibody (diluted 1:500; cat no. sc-492), rabbit anti-glutamate transporter-1 (GLT-1) polyclonal antibody (diluted 1:500; cat no. sc-15317) and mouse anti- $\beta$-actin monoclonal antibody (diluted 1:500; cat no. sc-376421). Membranes were then washed twice with tris-buffered saline with Tween-20 (TBST) for $20 \mathrm{~min}$. Subsequently, membranes were incubated with horseradish peroxidase conjugated anti-rabbit IgG (diluted 1:5,000; cat no. sc-2027) and anti-mouse IgG (diluted 1:5,000; cat no. sc-2025; both Santa Cruz Biotechnology, Inc.) for $2 \mathrm{~h}$ at room temperature. Membranes were washed four times with TBST for $40 \mathrm{~min}$. The immunoblot on the membrane was visualized using an enhanced chemiluminescence detection system (ImageQuant EC; GE Healthcare Life Sciences, 
Chalfont, UK) and densitometric signals were quantified using an imaging program (version 1.41; National Institutes of Health, Bethesda, MA, USA). Immunoreactive bands for all proteins were normalized to the corresponding bands for $\beta$-actin. The western blot results were analyzed with ImageJ Software (version 1.41; National Institutes of Health, Bethesda, MA, USA).

Multiplex cytokine ELISA. An immunosorbent assay was performed as described previously (16). The supernatant was then collected and total protein was determined by the Bradford method (16). The expression levels of inflammatory cytokines were quantified using ELISA kits specific for rats (cat no. 5000201; and Bio-Plex Pro ${ }^{\text {TM }}$ Rat Cytokine 24-plex Assay cat no. 171K1001M; Bio-Rad Laboratories, Inc.) according to the manufacturer's protocol. The cytokine contents in the brain samples were expressed as picograms of antigen per milligram of protein.

Statistical analysis. All data are presented as means \pm standard deviation. SPSS software (version 16.0; SPSS, Inc., Chicago, IL, USA) was used for the statistical analysis of all data. Statistical analysis was performed using one-way analysis of variance and followed by Student-Newman-Keuls post-hoc tests. $\mathrm{P}<0.05$ was considered to indicate a statistically significant difference.

\section{Results}

17-AAG attenuates cerebral edema. Brain edema suppresses cerebral perfusion pressure and oxygenation in the brain, resulting in an elevation in intracranial pressure following TBI (17). The formation of edema is an important factor to secondary injury following TBI. In the present study, the wet-dry weight method was used to evaluate cerebral edema at 1, 3, 5 and 7 days. As displayed in Fig. 1, the brain water content in the 17-AAG treatment group was significantly lower on days 1, 3 and 5 compared with the vehicle group $(\mathrm{P}<0.05)$.

17-AAG attenuates motor deficits. Recovery of motor deficits are expressed as $\Delta$ NSS in the present study. Fig. 2 revealed the changes in functional recovery at 1, 3, 5 and 7 days. It is evident that post-injury administration of 17-AAG significantly improved $(\mathrm{P}<0.05)$ the motor function recovery between 1 and 7 days following trauma compared with untreated rats.

17-AAG increases neuronal survival in the cortex. The cortex region of the brains was collected and immunostaining with the NeuN neuronal marker $24 \mathrm{~h}$ after TBI. Neuronal survival was quantified by counting the number of NeuN-positive cells in $1 \mathrm{~mm}$ of the rat cortex region. As presented in Fig. 3 , $24 \mathrm{~h}$ after TBI treatment with 17-AAG significantly increased neuronal survival in the cortex region of the brain following trauma $(\mathrm{P}<0.05)$.

17-AAG induces Hsp70 expression in the cortex. The expression levels of Hsp70 in the cortex at the $24 \mathrm{~h}$ time-point were measured by immunohistochemistry and western blotting. As depicted in Fig. 4, administration of 17-AAG produced significant elevations in Hsp70 protein expression levels $24 \mathrm{~h}$ following TBI, as compared with the untreated group $(\mathrm{P}<0.05)$.

17-AAG induces significant reductions in multiplex cytokine levels in the cortex. The expression levels of pro-inflammatory cytokines (IL-1 $\beta$, IL-6, and TNF- $\alpha$ ) in the cortex region of the rats were measured using commercially available ELISA kits at $24 \mathrm{~h}$ (Fig. 5). All three cytokine expression levels exhibited significant decreases following 17-AAG treatment compared with the vehicle group $(\mathrm{P}<0.05)$.

17-AAG attenuates neuronal apoptosis death in the cortex. The expression levels of caspase- 3 and $\mathrm{Bcl}-2$ in the cortex at $24 \mathrm{~h}$ were assessed by western blotting (Fig. 6). As demonstrated in Fig. 6, caspase-3 expression levels in the 17-AAG treatment group exhibited significant decreases compared with the vehicle group $(\mathrm{P}<0.05)$. Conversely Bcl-2 expression levels were significantly elevated $(\mathrm{P}<0.05)$. The present results indicate that $17-\mathrm{AAG}$ treatment leads to the reduction of neuronal apoptosis in the cortex following TBI.

17-AAG induces no significant changes in GLT-1 expression in the cortex of the brain. The protein expression levels of GLT-1 in the cortex were analyzed by western blotting $24 \mathrm{~h}$ after TBI (Fig. 7). Treatment with 17-AAG induced no significant changes in GLT-1 expression compared with the vehicle group $(\mathrm{P}>0.05)$.

\section{Discussion}

TBI is the primary cause of mortality and disability in young adults living in China (18). In the present study, a CCI model of brain trauma was established. The advantages of a CCI model include the ability to control deformation parameters, such as depth and velocity of the impact (19). Additionally, this model is able to imitate the spectrum of diffuse axonal injury and focal-type damage observed in TBI (19). In the present study, any rats that did not display moderate to severe neurological deficits following the surgery were excluded from further investigations.

Hsps are a family of chaperones that control the synthesis, folding and degradation of proteins within cells (20). The Hsp family constitutes a major control system for protein quality. Previous studies have demonstrated that overexpression of chaperones may attenuate the formation of protein aggregates in transgenic mouse models, thus reducing oxidative stress levels in addition to enhancing the antioxidant activity of enzymes (20-23). Furthermore, studies have revealed that the overexpression of Hsp70 may exert neuroprotective effects in animal models of neurological diseases $(22,23)$. As 17-AAG is an Hsp70 activator in vivo and displays little hepatotoxicity (24), the present study hypothesized that 17-AAG may be a promising approach for the treatment of TBI. The results of the present study demonstrated that administration of 17-AAG immediately following TBI significantly reduced brain edema and motor neurological deficits, in addition to increasing neuronal survival. Furthermore, the aforementioned findings were associated with the downregulation of pro-inflammatory cytokines. Previous studies have demonstrated that 17-AAG provides neuroprotective effects in various animal models 


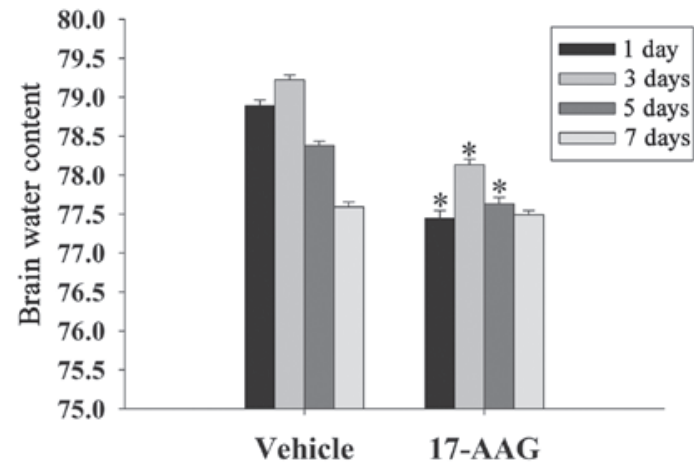

Figure 1. Effect of 17-AAG treatment on brain edema. Brain water content was determined at 1,3,5 and 7 days following TBI. Error bars represent the mean \pm standard error ( $\mathrm{n}=5$ per group). 17 -AAG significantly decreased brain edema 1,3 and 5 days following TBI ("P $<0.05$, vs. the vehicle group). TBI, traumatic brain injury; 17-AAG, 17-allylamino-demethoxygeldanamycin.

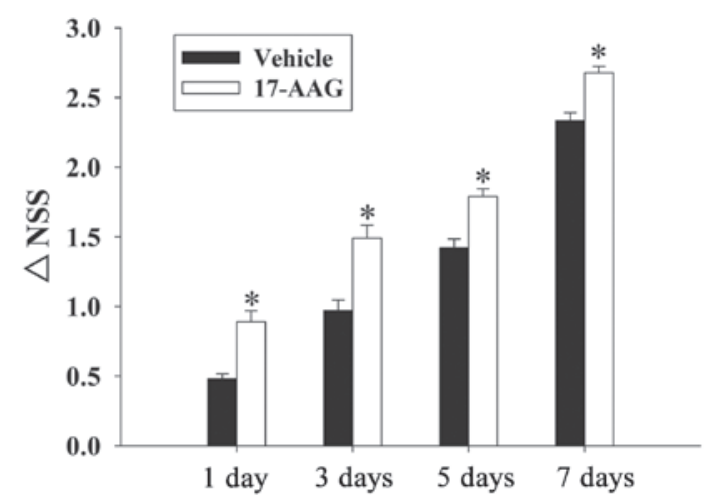

Figure 2. Effect of 17-AAG treatment on traumatic brain injury-induced motor deficits. The temporal changes in motor recovery were determined at $1,3,5$ and 7 days following TBI and calculated as $\triangle$ NSS. Error bars represent the mean \pm standard error ( $\mathrm{n}=5$ per group). 17 -AAG treatment significantly improved motor function at each time point ( $\mathrm{P}<0.05$, vs. the vehicle group). $\mathrm{TBI}$, traumatic brain injury; 17-AAG, 17-allylamino-demethoxygeldanamycin; NSS, Neurologic Severity Score.

of neurological diseases, including neurodegenerative disorders, epilepsy, ischemia and acute brain injury $(13,25)$. The results of the present study were concordant with those of the previous studies and consolidated upon previous evidence to demonstrate that post-injury treatment of 17-AAG provides neuroprotective properties through the attenuation of proinflammatory cytokines in a rat CCI model.

The inflammatory response induced following TBI is an important therapeutic target for reducing tissue damage following trauma (26). Additionally, it is a major contributing factor to secondary injury in TBI (26). A previous investigation revealed that when Hsp70 is overexpressed, the expression levels of the inducible isoform of nitric oxide synthase are reduced in astrocytes, whereas NF- $\mathrm{KB}$ is activated (27). Furthermore, in an in vitro study, Hsp70 prevented the lipopolysaccharide-induced upregulation of proinflammatory cytokines (28). Additionally, in a rat model of experimental stroke, overexpression of Hsp70 inhibited the production of IL-1 $\beta$ and TNF- $\alpha$ (29). The present study did not establish the existence of a direct association between TBI-induced functional deficits and pro-inflammatory factors, however, in vivo evidence that $17-\mathrm{AAG}$ can provide a neuroprotective effect
A

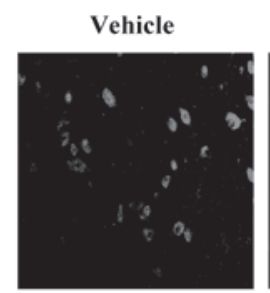
17-AAG

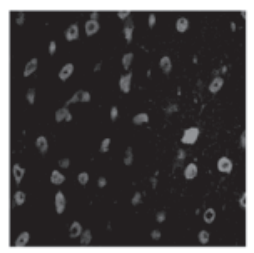

B

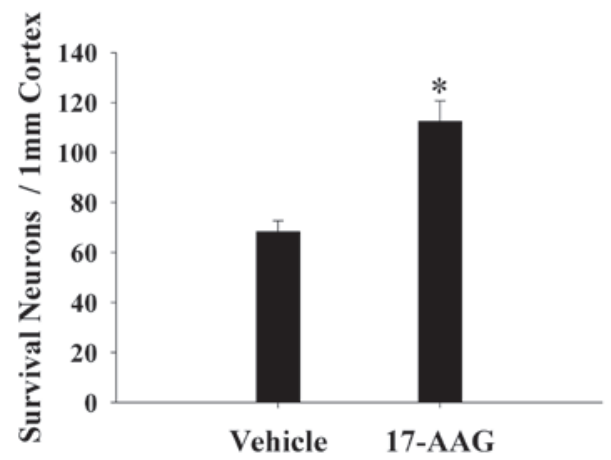

Figure 3. Neuronal survival was quantified using (A) Neuron-specific nuclear protein immunostaining in the cortex $24 \mathrm{~h}$ following traumatic brain injury. Magnification, x100. (B) Quantitative summary of the data reveals the number of surviving neurons/1 mm length of cortex sectors. Error bars are presented as means \pm standard error ( $n=5$ per group). The results demonstrate that administration of 17-AAG significantly increases neuronal survival in the cortex ( $\mathrm{P}<0.05$, vs. vehicle group). 17-AAG, 17-allylamino-demethoxygeldanamycin.

A

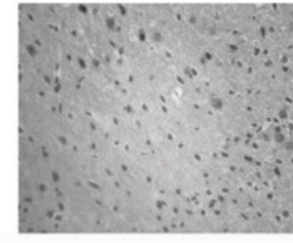

Vehicle

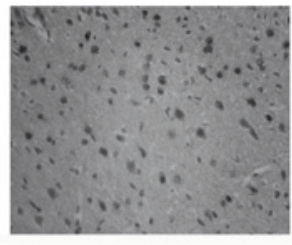

17-AAG
B

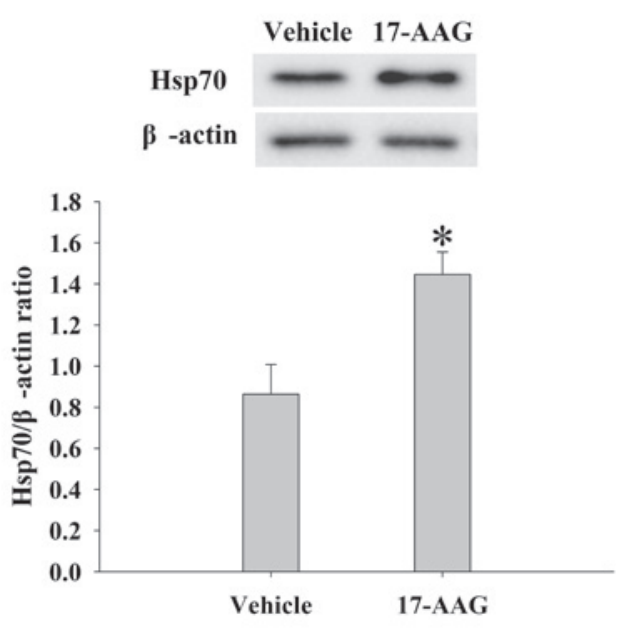

Figure 4. Effect of 17-AAG on Hsp70 expression levels following traumatic brain injury. (A) The immunohistochemistry images of Hsp70 in the vehicle and 17-AAG groups at $24 \mathrm{~h}$. Magnification, x40. (B) Western blot analysis of the expression levels of Hsp70 in the cortex of the rats at $24 \mathrm{~h}$. The quantitative results of Hsp70 were expressed as the ratio of densitometries of Hsp70 to $\beta$-actin bands. The error bars represent the mean \pm standard error $(n=5$ per group). The results indicate significant upregulation of Hsp70 expression levels in the 17-AAG group ( $\mathrm{P}<0.01$, vs. the vehicle group). TBI, traumatic brain injury; 17-AAG, 17-allylamino-demethoxygeldanamycin; HSP70, heat shock protein. 

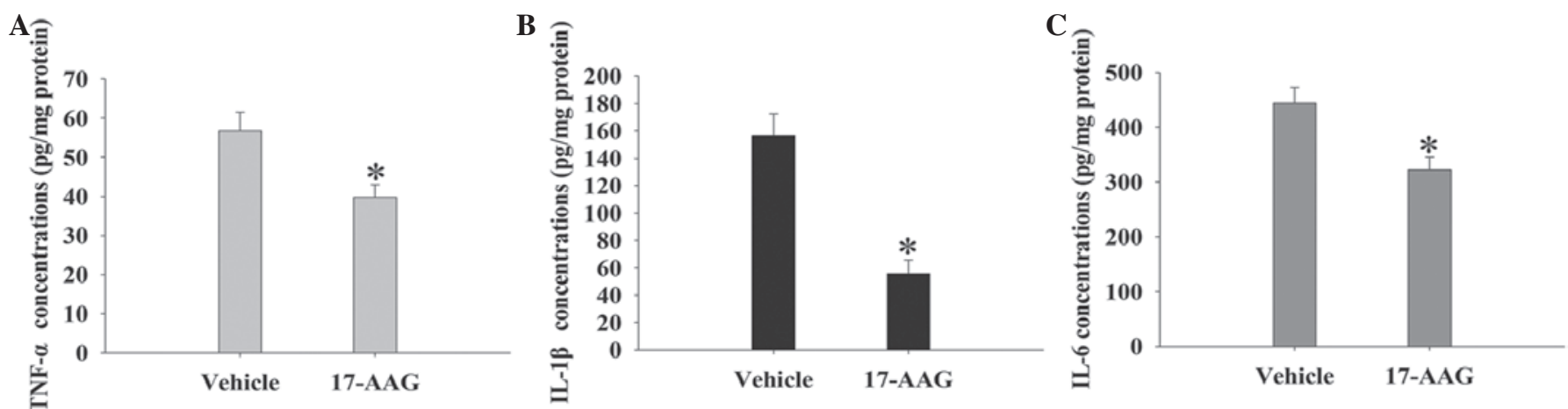

Figure 5. Cytokine expression levels in rats from the vehicle and 17-AAG groups with respect to (A) TNF- $\alpha$, (B) IL-1 $\beta$ and (C) IL-6. The error bars represent the means \pm standard error ( $\mathrm{n}=5$ per group). ${ }^{*} \mathrm{P}<0.05$, vs. vehicle group. 17-AAG, 17-allylamino-demethoxygeldanamycin; IL, interleukin; TNF- $\alpha$, tumor necrosis factor- $\alpha$.

A
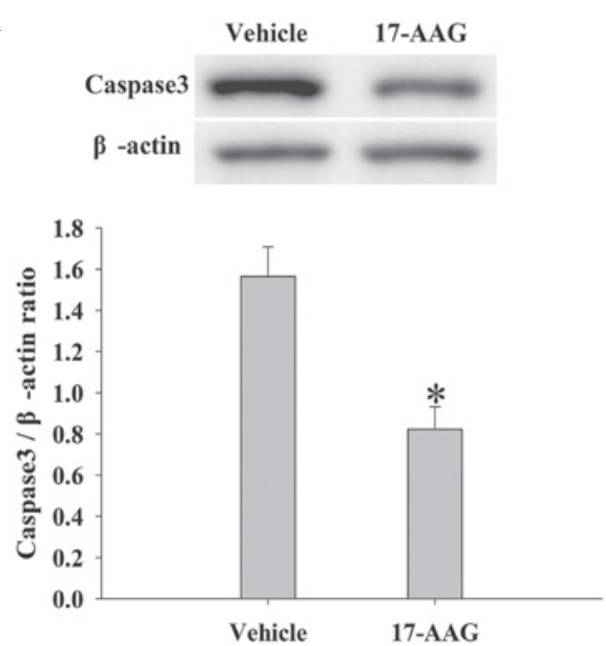

B
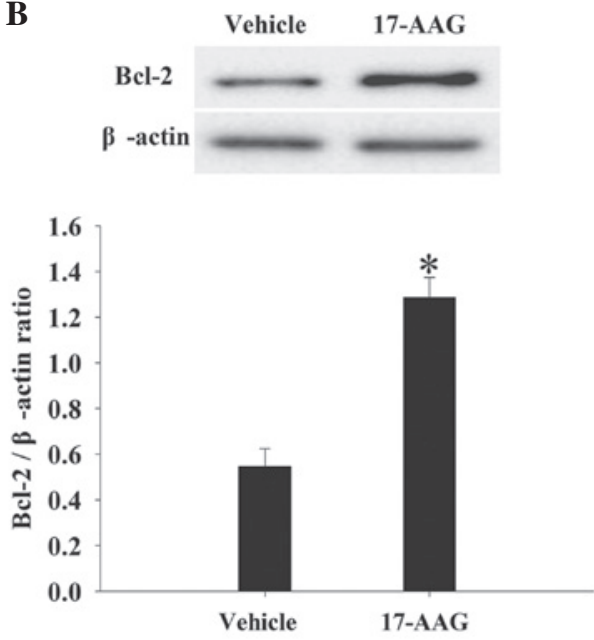

Figure 6. (A) Western blot analysis of the expression levels of caspase-3 in the cortex of rats $24 \mathrm{~h}$ following TBI. (B) Western blot analysis of the expression levels of Bcl- 2 in the cortex of rats $24 \mathrm{~h}$ following TBI. The quantitative results of caspase- 3 and Bcl- 2 were expressed as the ratio of densitometries with $\beta$-actin bands. The error bars represent the means \pm standard error ( $n=5$ per group). 17-AAG caused a significant decrease in caspase-3, in addition to marked elevation in Bcl-2 expression levels ( $\mathrm{P}<0.01$, vs. the vehicle group). 17-AAG, 17-allylamino-demethoxygeldanamycin; Bcl-2, B-cell lymphoma-2; TBI, traumatic brain injury.

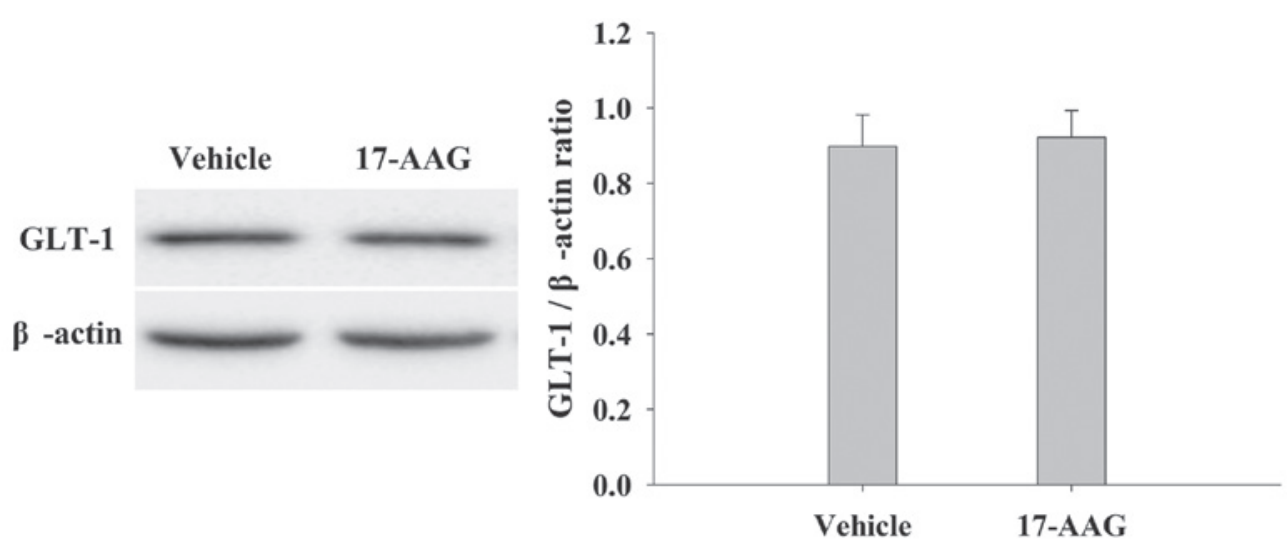

Figure 7. Western blot analysis of the expression levels of GLT-1 in the cortex of the rats in the vehicle and 17-AAG groups at 24 h. Quantitative results of GLT-1 were expressed as the ratio of densitometries of GLT-1 to $\beta$-actin bands. The error bars represent the means \pm standard error ( $\mathrm{n}=5$, per group). The results demonstrate that 17 -AAG causes significant changes in GLT-1 expression ( $\mathrm{P}>0.05$, vs. the vehicle group). GLT-1, glutamate transport-1; 17-AAG, 17-allylamino-demethoxygeldanamycin.

partly by inhibiting proinflammatory cytokine activation (8) has been demonstrated.

Excitotoxicity is another crucial process in nervous cell death following TBI (30). Astrocytes may protect against glutamate excitotoxicity via glutamate transporters, and GLT-1 is the most important glutamate transporter in the brains of rats (31). However, in the present study, no significant change in GLT-1 expression levels following post-TBI 17-AAG 
treatment were observed. It was there hypothesized that the neuroprotective effect of 17-AAG is not associated with the regulation of GLT-1 protein expression.

In summary, the present study demonstrated that the administration of Hsp70 activator 17-AAG reduced brain edema and motor neurological deficits, in addition to increasing neuronal survival, in a rat model of TBI. Furthermore, 17-AAG reduced TNF- $\alpha$, IL- $1 \beta$ and IL- 6 protein expression levels. Conversely, no significant changes in GLT-1 expression levels were observed. The findings of the present study emphasize that $17-\mathrm{AAG}$ provides neuroprotection via anti-inflammatory effects in a rat model of TBI.

\section{References}

1. Hyder AA, Wunderlich CA, Puvanachandra P, Gururaj G and Kobusingye OC: The impact of traumatic brain injuries: A global perspective. NeuroRehabilitation 22: 341-353, 2007.

2. Johnson VE, Stewart W and Smith DH: Widespread $\tau$ and amyloid- $\beta$ pathology many years after a single traumatic brain injury in humans. Brain Pathol 22: 142-149, 2012.

3. Werner $\mathrm{C}$ and Engelhard K: Pathophysiology of traumatic brain injury. Br J Anaesth 99: 4-9, 2007.

4. Loane DJ and Faden AI: Neuroprotection for traumatic brain injury: Translational challenges and emerging therapeutic strategies. Trends Pharmacol Sci 31: 596-604, 2010.

5. Kamm K, Vanderkolk W, Lawrence C, Jonker M and Davis AT: The effect of traumatic brain injury upon the concentration and expression of interleukin- $1 \beta$ and interleukin-10 in the rat. J Trauma 60: 152-157, 2006.

6. Aibiki M, Maekawa S, Ogura S, Kinoshita Y, Kawai N and Yokono S: Effect of moderate hypothermia on systemic and internal jugular plasma IL-6 levels after traumatic brain injury in humans. J Neurotrauma 16: 225-232, 1999.

7. Hang C-H, Shi J-X, Li J-S, Li W-Q and Wu W: Expressions of intestinal NF-kappaB, TNF- $\alpha$, and IL-6 following traumatic brain injury in rats. J Surg Res 123: 188-193, 2005.

8. Chen G, Shi JX, Hang CH, Xie W, Liu J and Liu X: Inhibitory effect on cerebral inflammatory agents that accompany traumatic brain injury in a rat model: A potential neuroprotective mechanism of recombinant human erythropoietin (rhEPO). Neurosci let 425: 177-182, 2007.

9. Mayer MP and Bukau B: Hsp70 chaperones: Cellular functions and molecular mechanism. Cell Mol Life Sci 62: 670-684, 2005.

10. Nowak TS Jr and Jacewicz M: The heat shock/stress response in focal cerebral ischemia. Brain Pathol 4: 67-76, 1994.

11. Kelly S and Yenari MA: Neuroprotection: Heat shock proteins. Curr Med Res Opin 18 (Suppl 2): s55-s60, 2002

12. Lee S-H, Kim M, Yoon B-W, Kim YJ, Ma SJ, Roh JK, Lee JS and Seo JS: Targeted hsp70. 1 disruption increases infarction volume after focal cerebral ischemia in mice. Stroke 32: 2905-2912, 2001.

13. Kim N, Kim JY and Yenari MA: Anti-inflammatory properties and pharmacological induction of Hsp70 after brain injury. Inflammopharmacology 20: 177-185, 2012.

14. Chen SF, Hsu CW, Huang WH and Wang JY: Post-injury baicalein improves histological and functional outcomes and reduces inflammatory cytokines after experimental traumatic brain injury. Br J Pharmacol 155: 1279-1296, 2008.

15. Cui CM, Gao JL, Cui Y, Sun LQ, Wang YC, Wang KJ, Li R, Tian YX and Cui JZ: Chloroquine exerts neuroprotection following traumatic brain injury via suppression of inflammation and neuronal autophagic death. Mol Med Rep 12: 2323-2328, 2015.
16. Wei J, Pan X, Pei Z, Wang W, Qiu W, Shi Z and Xiao G: The beta-lactam antibiotic, ceftriaxone, provides neuroprotective potential via anti-excitotoxicity and anti-inflammation response in a rat model of traumatic brain injury. $\mathrm{J}$ Trauma Acute Care Surg 73: 654-660, 2012.

17. Ikeda Y and Long DM: The molecular basis of brain injury and brain edema: The role of oxygen free radicals. Neurosurgery 27: 1-11, 1990.

18. McKinlay A, Grace RC, Horwood LJ, Fergusson DM, Ridder EM and MacFarlane MR: Prevalence of traumatic brain injury among children, adolescents and young adults: Prospective evidence from a birth cohort. Brain Inj 22: 175-181, 2008.

19. O'Connor WT, Smyth A and Gilchrist MD: Animal models of traumatic brain injury: A critical evaluation. Pharmacol Ther 130: 106-113, 2011.

20. Outeiro TF, Klucken J, Strathearn KE, Liu F, Nguyen P, Rochet JC, Hyman BT and McLean PJ: Small heat shock proteins protect against $\alpha$-synuclein-induced toxicity and aggregation. Biochem Biophys Res Commun 351: 631-638, 2006.

21. Adachi H, Katsuno M, Minamiyama M, Sang C, Pagoulatos G, Angelidis C, Kusakabe M, Yoshiki A, Kobayashi Y, Doyu M and Sobue G: Heat shock protein 70 chaperone overexpression ameliorates phenotypes of the spinal and bulbar muscular atrophy transgenic mouse model by reducing nuclear-localized mutant androgen receptor protein. J Neurosci 23: 2203-2211, 2003.

22. Hollander JM, Martin JL, Belke DD, Scott BT, Swanson E, Krishnamoorthy V and Dillmann WH: Overexpression of wild-type heat shock protein 27 and a nonphosphorylatable heat shock protein 27 mutant protects against ischemia/reperfusion injury in a transgenic mouse model. Circulation 110: 3544-3552, 2004.

23. Hutter JJ, Mestril R, Tam EK, Sievers RE, Dillmann WH and Wolfe CL: Overexpression of heat shock protein 72 in transgenic mice decreases infarct size in vivo. Circulation 94: 1408-1411, 1996.

24. Fumo G, Akin C, Metcalfe DD and Neckers L: 17-Allylamino-17-demethoxygeldanamycin (17-A AG) is effective in down-regulating mutated, constitutively activated KIT protein in human mast cells. Blood 103: 1078-1084, 2004.

25. Harrison EM, Sharpe E, Bellamy CO, McNally SJ, Devey L, Garden OJ, Ross JA and Wigmore SJ: Heat shock protein 90-binding agents protect renal cells from oxidative stress and reduce kidney ischemia-reperfusion injury. Am J Physiol Renal Physiol 295: F397-F405, 2008.

26. Morganti-Kossmann MC, Rancan M, Stahel PF and Kossmann T: Inflammatory response in acute traumatic brain injury: A double-edged sword. Curr Opin Crit Care 8: 101-105, 2002.

27. Heneka MT, Sharp A, Klockgether T, Gavrilyuk V and Feinstein DL: The heat shock response inhibits NF-kappaB activation, nitric oxide synthase type 2 expression, and macrophage/microglial activation in brain. J Cereb Blood Flow Metab 20: 800-811, 2000.

28. Ding XZ, Fernandez-Prada CM, Bhattacharjee AK and Hoover DL: Overexpression of hsp-70 inhibits bacterial lipopolysaccharide-induced production of cytokines in human monocyte-derived macrophages. Cytokine 16: 210-219, 2001

29. Zheng Z, Kim JY, Ma H, Lee JE and Yenari MA: Anti-inflammatory effects of the $70 \mathrm{kDa}$ heat shock protein in experimental stroke. J Cereb Blood Flow Metab. 28: 53-63, 2008.

30. Palmer AM, Marion DW, Botscheller ML, Swedlow PE, Styren SD and DeKosky ST: Traumatic brain injury-induced excitotoxicity assessed in a controlled cortical impact model. J Neurochem 61: 2015-2024, 1993

31. Tanaka K, Watase K, Manabe T, Yamada K, Watanabe M, Takahashi K, Iwama H, Nishikawa T, Ichihara N, Kikuchi T et al: Epilepsy and exacerbation of brain injury in mice lacking the glutamate transporter GLT-1. Science 276: 1699-1702, 1997. 